\title{
NUMERICAL SIMULATIONS OF STREAMWISE VORTICES ON A HIGH- LIFT WING
}

\author{
T. Landa*, R. Radespiel ${ }^{+}$and J. Wild ${ }^{\ddagger}$
}

\begin{abstract}
This contribution presents results of numerical simulations on a generic high-lift configuration. Properties of the computational grid are briefly described. The numerical simulations are performed with the DLR-TAUCode at different angles of attack up to stall. The Menter-SST eddy viscosity turbulence model and the JHhv2 Reynolds-Stress-Model are applied. Streamwise vortices arise at the edge of a slat, which is cut off in spanwise direction, and the corresponding edge of the clean nose. These vortices interact with the flow along the suction side of the wing. While proceeding downstream, a strong interaction between the vortices is observed for high angles of attack. The behavior of the vortex system and the influence on the high-lift performance of the configuration is characterized. In particular, the effect of the applied turbulence models of different types on the prediction of the vortex behavior is shown within this contribution.
\end{abstract}

\section{Keywords}

High-Lift Aerodynamics, Longitudinal Vortices, Computational Fluid Dynamics, Turbulence Modeling

\section{NOMENCLATURE}

b span, m

$\mathrm{C}_{\mathrm{D}} \quad$ drag coefficient, -

$\mathrm{C}_{\mathrm{L}} \quad$ lift coefficient, -

$\mathrm{C}_{\text {ref }}$ reference chord length (flaps retracted), $\mathrm{m}$

Ma Mach number, -

Re Reynolds number, -

$\mathrm{t} \quad$ time, $\mathrm{s}$

$t_{\text {conv }} \quad$ convective time unit, $s$

$V_{\infty} \quad$ freestream velocity, $\mathrm{m} / \mathrm{s}$

$\mathrm{x}, \mathrm{y}, \mathrm{z}$ cartesian coordinates

$\mathrm{y}^{+} \quad$ dimensionless wall distance, -

a angle of attack, 。

$\eta \quad$ relative spanwise position $(\eta=y / b),-$

$\Gamma \quad$ circulation, $\mathrm{m}^{2} / \mathrm{s}$

$\Delta \mathrm{s}_{\text {init }} \quad$ initial wall spacing, $\mathrm{mm}$

$\varphi \quad$ sweep angle, ${ }^{\circ}$

$\omega \quad$ vorticity, $1 / \mathrm{s}$

\section{Subscript}

$\mathrm{n}$ normal to leading edge

\section{INTRODUCTION}

Modern aircraft operate within a large range of velocities. To obtain the required lift at low speeds during take-off and landing, the lift coefficient is increased by high-lift devices such as leading edge and trailing edge devices. Nowadays, common high-lift systems consist of a single slotted slat (leading edge) and a single slotted Fowler flap (trailing edge) [1]. Compared to multi-slotted high-lift devices, such a configuration is a good compromise between aerodynamic high-lift performance and the complexity and the weight of the high-lift system. The basic aerodynamic effects of slotted multi-element high-lift configurations are well understood [2]. Nevertheless, the prediction of maximum lift of realistic configurations requires to consider details of the three-dimensional highlift device.

\begin{abstract}
A significant influence on the aerodynamic behavior is observed due to (underwing) engine integration. At the position of the engines pylon, the slat is cut out. At the spanwise end faces of the intercepted slat and at the corresponding faces of the clean-nose part above the pylon vortices are induced. Moreover, the flow around the engine causes two additional vortices. The result is a complex system consisting of different vortices trailing downstream close to the suction side of the wing. The vortex system strongly influences the flow behavior in this region, which generally leads to a significant loss of maximum achievable lift [3]. These losses can be compensated by a nacelle strake, which is mounted at the engine. The nacelle strake causes a particular vortex. This vortex positively influences the flow field downstream causing an increase of maximum lift.
\end{abstract}

In recent years much effort has been taken to investigate the effects of vortex systems on the high-lift behavior of realistic configurations. Aiming at a better understanding of the complex vortex behavior, numerous experimental and numerical investigations have been performed in various research projects. Important contributions in this area of research are provided within the European projects EUROLIFT and EUROLIFT II [4]-[8]. These projects include extensive numerical and experimental studies of different high-lift configurations with different levels of complexity, which provides a deep understanding of the influence of different components on the lift and drag behavior. Particularly the effects on maximum lift were analyzed. Another remarkable work is the research project HINVA (High-Lift INflight Validation) [9]. Within this project, numerical and experimental investigations with detailed models of an Airbus A320 and large-scale flight tests have been performed, to improve the prediction of the aerodynamic performance at maximum lift. However, the prediction of the aerodynamic effects at maximum lift

\footnotetext{
${ }^{*}$ Research Assistant, Institute of Fluid Mechanics, TU Braunschweig, Hermann-Blenk-Str. 37, 38108 Braunschweig, mailto: t.landa@tu-braunschweig.de, Phone: +49 53139194268

${ }^{\dagger}$ Professor and Head of Institute, Institute of Fluid Mechanics, TU Braunschweig, Hermann-Blenk-Str. 37, 38108 Braunschweig

${ }^{\ddagger}$ Research Scientist and Team Lead High-Lift, DLR Institute of Aerodynamics and Flow Technology, Lilienthalplatz 7, 38108 Braunschweig
} 
including the behavior of the vortex system with numerical methods is still a challenging task [10]. Similar findings derive from the NASA High-lift prediction workshop. The main focus was to predict the correct flow behavior with modern numerical methods at a generic three-element high-lift configuration without slat interception and engine. At this configuration a vortex system in streamwise direction occurs at the wing tip. Results of various numerical simulations showed significant differences compared to experimental results [11]. Particularly, the behavior of the streamwise vortex system differed. It was shown that the applied turbulence model has a large influence on the prediction of the behavior of the vortex system [12].

Further studies dealt with the influence of different turbulence models on the prediction of streamwise vortices by analyzing the tip vortex of a half-wing mode without high-lift devices. Significant differences in the behavior of the vortex occur. Simulations with eddy viscosity models show a rapid decay of the vortex which does not correspond to experimental data. In contrast, the decay of the streamwise vortex predicted with Reynolds Stress Models is significantly lower. The results with Reynolds Stress Models (RSM) are in good agreement with experimental data [13][14].

To approach a better prediction of the vortex systems and their influence on wing stall, in this contributions the vortex system at a slat end is investigated in a generic setup. Particularly, the effect of the applied turbulence model on the behavior of the vortex system is of great interest. For the fundamental studies herein, we use a simplified configuration, which is a generic high-lift configuration without an engine. The slat is finite, representing half of a slat cut-out for engine integration on commercial aircraft. With this geometry, it is possible to investigate one part of the vortex system in detail, which is still relevant for realistic configurations. A detailed description of the geometry follows in section 2.2 .

Recently, the authors presented results of simulations with the Menter-SST turbulence model [15]. Based on these results, the computational grid has been improved. With this modified grid, simulations with the Menter-SST eddy viscosity model and the JHh-v2 RSM turbulence model are performed. The results of these simulations are presented in this contribution. The investigations aim at a deeper understanding of the interaction between longitudinal vortices and the suction side of the high-lift wing at different angles of attack. Particularly the effects on stall behavior and maximum lift are of great interest. The numerical methods, the geometry and the computational grid used for the simulations herein are described in detail in the first part of this contribution. In addition, an overview of the performed simulations is given. The results are presented and discussed. The global flow field as well as the characteristics of the vortex system are shown. In the last part, the results are summarized and an outlook to future work is given.

\section{NUMERICAL SETUP}

\subsection{Methods}

For the simulations presented in this contribution the DLR TAU-Code (Release 2014.2.0) is used as flow solver [16][17]. With this software package, it is possible to compute flow fields on structured, unstructured or hybrid grids by solving the Reynolds-Averaged-Navier-Stokes equations based on a finite volume method. To compute the fluxes, central as well as different upwind spatial discretization schemes are available. For time-stepping either a semi-implicit LU-SGS scheme or explicit RungeKutta schemes can be applied. The TAU-Code offers different convergence acceleration techniques like local time stepping, residual smoothing and a multigrid approach. In order to accelerate the numerical solution process at nearly incompressible flow conditions, low Mach number preconditioning is applied. For the simulations presented in this contribution, the central discretization scheme to calculate fluxes and the LU-SGS scheme for time stepping were used. The previously described acceleration techniques have been applied.

To simulate turbulent flows, the TAU-Code follows the Reynolds-Averaged-Navier-Stokes (RANS) approach combined with a turbulence model. Various turbulence models of different types are implemented. Here, the twoequation Menter-SST (Shear Stress Transport) and the JHh-v2 (Jakirlić Hanjalić homogeneous) turbulence models are applied. The Menter-SST model [18] is based on the hypothesis of Boussinesq and belongs to the class of eddy viscosity models. Although the ability of these models to accurately simulate vortices is known to be limited [13][14], the robustness of this model is of great advantage to create a fundamental understanding of the flow around this geometry. Furthermore, the results serve as a basis for comparison with the applied Reynolds Stress Model. The JHh-v2 RSM model follows a secondmoment closure approach, which means that a transport equation is solved for each component of the Reynolds stress tensor. This model is based on the $\mathrm{JHh}$ turbulence model developed by Jakirlić and Hanjalić [19] and has been extended by Probst [20]. Recent work shows promising results in vortex prediction using the $\mathrm{JHh}-\mathrm{v} 2$ turbulence model [14][21]

\subsection{Geometry}

The generic three-dimensional configuration is based on the DLR F15 high-lift airfoil. In recent years, this airfoil has been extensively investigated both in experimental and numerical studies and thus a high level of knowledge is achieved [21]-[22]. The following parameters define the position of the slat and the Fowler flap related to the airfoil geometry normal to the leading edge.

- Slat: $28.8^{\circ}$ deflection, $2.09 \%$ overlap, $2.61 \%$ gap

- Fowler flap: $30.3^{\circ}$ deflection, $1.52 \%$ overlap, $0.968 \%$ gap

The generic high-lift configuration is a swept wing $(\varphi=$ $25^{\circ}$ ) without twist and dihedral. The span is $b=1.7525 \mathrm{~m}$ and the chord is constant for the whole wing. With retracted flaps, the chord normal to the leading edge is $c_{n}$ $=0.6 \mathrm{~m}$. This value corresponds to a chord in streamwise direction of $c_{\text {ref }}=0.662 \mathrm{~m}$, which is taken as the reference 
chord length for the evaluation. At the inner part of the wing $(\eta=0 \%-62.6 \%)$, the slat is retracted resulting in a two-element configuration with a clean. Outboard, the slat is extended with the geometric parameters mentioned above. The flap is extended along the whole span. At the spanwise end, the wing is cut off in line of flight and no additional wingtip-device is mounted. Figure 1 illustrates the geometry of the three-dimensional high-lift configuration.

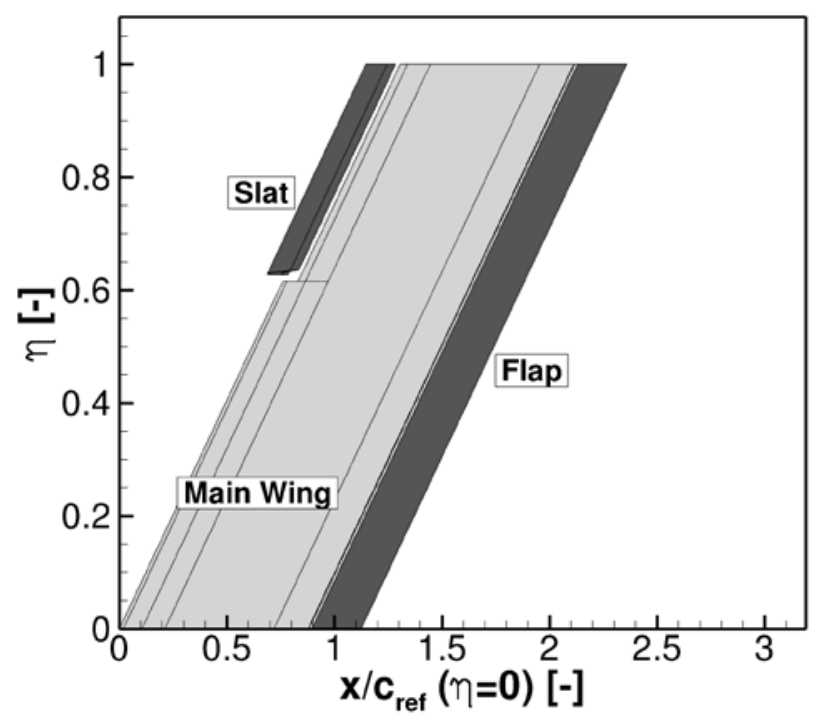

Fig. 1 Top view on the high-lift configuration

\subsection{Grids}

For the generation of the computational grids the commercial software Gridgen (V 15.18, Pointwise, Inc.) has been used. The reference grid is a hybrid grid consisting of structured and unstructured parts. The surface grid contains mostly structured areas, only the spanwise end faces of slat, wing and the Fowler flap are discretized with an unstructured surface grid. The structured volume part of the grid consists of 60 layers to ensure a good resolution of the boundary layers by structured cells. The initial wall spacing is $\Delta$ sinit $=0.003$ $\mathrm{mm}$ such that dimensionless wall distances of $\mathrm{y}^{+}<1$ are obtained largely, which is required to accurately predict the boundary layer. Only in regions of suction peaks or strongly vortex influenced regions $\mathrm{y}^{+}$-values are slightly greater than 1 . The expansion rate in wall-normal direction within the structured layers to resolve the boundary layer is lower than 1.18 globally. Thus, the boundary layer is resolved with more than 35 cells at a chordwise position of $x / c_{\text {ref }}=0.1$ and more than 40 cells at a chordwise position of $x / c_{\text {ref }}=0.8$. The maximum aspect ratios close to the wall are $\left(\Delta x / \Delta s_{\text {init }}\right)_{\max } \approx 2000$ in chordwise direction and $\left(\Delta y / \Delta s_{\text {init }}\right)_{\max } \approx 3333$ in spanwise direction. The latter one occurs at an undisturbed position inboard at the wing. Within the vortex-influenced region, the spanwise aspect ratio is significantly lower $\left(\Delta y / \Delta s_{\text {init }} \approx 666\right)$. Structured blocks have also been used for the wake region behind the flap and in the slat and wing cove. In addition, the region downstream of the slat cut-off is also resolved with additional structured cells above the structured part to resolve the boundary layer. Here, a rectangular box with a constant cell spacing in $y$ - and $z$-direction is used to account for the presence of streamwise vortices. The box is directly connected to the structured cells above the surface. Hence, numerical smearing of vortices due to tetrahedral cells is minimized using equally spaced hexahedral cells. An improvement of capturing the behavior of vortices using a hexahedral box has already been shown by Eliasson et al. [4].

The whole computational domain has the shape of a hemisphere. Outside the previously described structured areas unstructured tetrahedral cells are used. The resolution decreases to the outer edge. The high-lift configuration is fixed at the plane of symmetry, which is assumed to be an Eulerian wall without viscous effects. The spherical farfield boundary has a distance of $100 \mathrm{~m}$ $\left(\approx 150^{*} C_{\text {ref }}\right.$ respectively $\left.\approx 58^{*} b\right)$ to the origin, which is located at the leading edge of the wing at $\eta=0$. In total the grid consists of about 60 million points.

In addition, two other computational grids were derived from the reference grid. The overall structure (structured parts, vortex box, computational domain) is maintained, whereas the spatial resolution varies between the grids. For the coarse grid, the number of cells in the structured part is halved in each direction $(x, y, z)$ and the unstructured part is adapted. Furthermore, a grid with a local refinement in the region of the vortices was created. RANS simulations with this grid provide information about the influence of the spatial discretization on the prediction of the vortices. Table 1 shows the spacing of the grids in the box to capture the vortices and the total number of grid points.

Tab. 1 Spacing of the hexahedral cells within the vortex box and total grid points of the different grids

\begin{tabular}{l|ccc}
\hline grid & $\Delta y / \Delta z$ & $\max . \Delta x$ & total points \\
\hline coarse & $4 \mathrm{~mm}$ & $11.5 \mathrm{~mm}$ & $\sim 8.2 \mathrm{mio}$. \\
\hline reference & $2 \mathrm{~mm}$ & $5.75 \mathrm{~mm}$ & $\sim 60$ mio. \\
\hline $\begin{array}{l}\text { locally } \\
\text { refined }\end{array}$ & $0.5 \mathrm{~mm}$ & $1 \mathrm{~mm}$ & $\sim 113$ mio. \\
\hline
\end{tabular}

\subsection{Flow Conditions}

The flow conditions and the model size correspond to common experimental settings of the DLR F15 airfoil. Various wind tunnel experiments [22]-[24] and numerical studies [21][24] have been performed at $\operatorname{Re}_{n}=2.096 \cdot 10^{6}$ and $\mathrm{Ma}_{\mathrm{n}}=0.15$. For the simulations performed at the swept wing $\left(\varphi=25^{\circ}\right)$ it is desired to have comparable flow conditions normal to the leading edge, where the twodimensional airfoil is present. With the following equations the freestream values of $\mathrm{Re}$ and $\mathrm{Ma}$ are calculated for the swept wing without dihedral at an angle of attack of $\alpha=6^{\circ}$ [25].

$$
\begin{aligned}
& \text { (1) } M a=\frac{M a_{n}}{\cos \varphi \sqrt{1+\sin ^{2} \alpha \cdot \tan ^{2} \varphi}} \\
& \text { (2) } \operatorname{Re}=\frac{\operatorname{Re}_{n}}{\cos ^{2} \varphi \sqrt{1+\sin ^{2} \alpha \cdot \tan ^{2} \varphi}}
\end{aligned}
$$


The resulting values of $\mathrm{Re}$ and $\mathrm{Ma}$ are assumed to be constant to have comparable conditions for all simulations although the component normal to the leading edge differs depending on the angle of attack. Simulations have been performed at different angles of attack with the following freestream conditions.

$$
\begin{aligned}
& \left.-R e=2.546 \cdot 10^{6} \text { (related to } c_{\text {ref }}=0.662 \mathrm{~m}\right) \\
& -M a=0.1653 \\
& -V_{\infty}=56.735 \mathrm{~m} / \mathrm{s} \\
& -\alpha=6^{\circ} / 8^{\circ} / 10^{\circ} / 12^{\circ} / 12.5^{\circ} / 13^{\circ} / 13.5^{\circ}
\end{aligned}
$$

Turbulent flow is assumed for all simulations performed with the Menter-SST turbulence model. The JHh-v2 mode requires defining transition positions. Based on the Menter-SST results, the location of transition on the lower surface is defined closely downstream of the stagnation line. The transition of the upper surface is set close to the suction peak of each component. These artificial transition positions do not necessarily represent the physical position, which can not be exactly determined in the absence of corresponding experiments. Nevertheless, the applied strategy to define the transition for the simulation is common practice in many applications of high Reynolds number flows. A detailed description how transition is modelled within the $\mathrm{JHh}-\mathrm{v} 2$ model can be found in [26]

The simulations with both turbulence models started at a $=6^{\circ}$ without a restart solution. Based on these solutions, the angle of attack has been increased gradually. In addition to the simulations with the steady solver, an unsteady simulation (URANS) with the JHh-v2 model has been performed at $\alpha=6^{\circ}$.

\subsection{Validation of the numerical approach}

DLR F-15 model has been extensively investigated with various experiments including different sweep angles, flap and slat settings and flow conditions. Unfortunately, a configuration with a slat cut-out or cut-off, where a vortex system interacts with the suction side has not been tested yet. Hence, no experimental reference data is available for a comparison with the presented simulations. Nevertheless, the applied turbulence models have been tested on a related test case. Cécora investigated the ability of different turbulence models to predict streamwise vortices on a NACA 0012 half-wing model [27]. At this configuration a streamwise vortex develops at the tip of the inclined wing $\left(\alpha=10^{\circ}\right)$. The computational grid consists of $\mathbf{1 3 . 2}$ million grid points in total. Again, structured blocks with hexahedral cells are used to capture the vortex accurately. The simulations were performed at a Mach number of $\mathrm{Ma}=0.16$ and a Reynolds number of $\mathrm{Re}=$ $4.35 \times 10^{6}$. Cécora used different turbulence models and compared the predicted vortices with experimental data at the same conditions. Figure 2 shows the streamwise velocity component of the vortex core above and behind the NACA 0012 half-wing.

In the phase of axial acceleration on the wing $\left(x_{a} / c<0\right)$, the applied eddy viscosity models (Menter-SST and SAO) underestimate the core velocity. The Reynolds Stress Models (JHh-v2 model with linear or quadratic redistribution term) predict core velocities, which are in good agreement with experimental data. Furthermore, the dissipation of the vortex behind the wing $\left(x_{a} / c>0\right)$ differs strongly among the applied turbulence models. Both eddy viscosity models show a strong decay of the axial velocity behind the trailing edge of the wing. In contrast, the Reynolds Stress Models predict a slow decrease of the core velocity, which again matches the experimental data. One finds that the Reynolds Stress Models capture the observed vortex behavior while the eddy viscosity models show a rapid dissipation of the vortex.

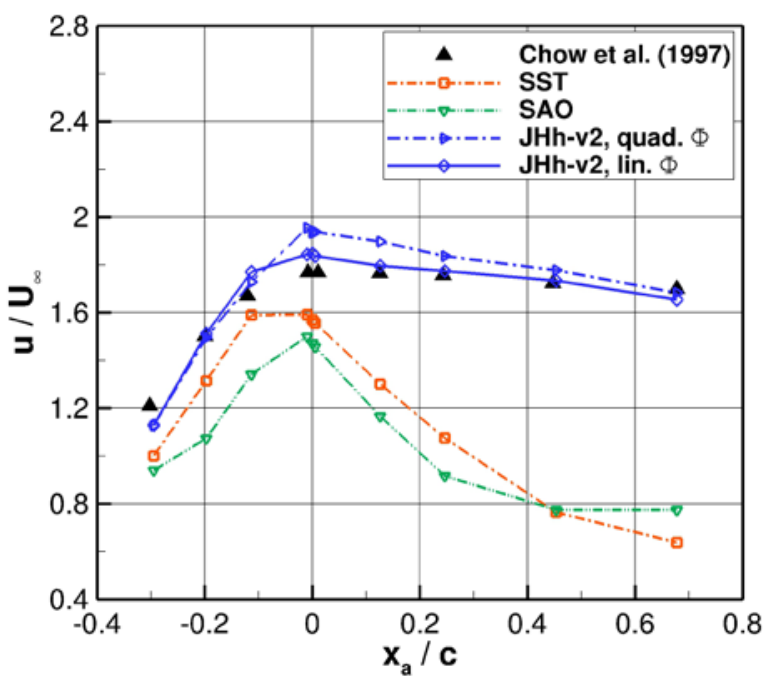

Fig. 2 NACA 0012 half-wing. Streamwise velocity component along the vortex core [27]

With the present work, the findings of Cécora are transferred to a more complex, practically-relevant test case. Again, the effect of the applied turbulence model on the vortex prediction is investigated. Based on the results of Cécora, the JHh-v2 RSM model with a linear redistribution term is used here. As an eddy viscosity model, the established Menter-SST model is applied (q.v. 2.1.)

\section{RESULTS}

This section presents the results of the simulations on the high-lift configuration. The convergence behavior of the simulations and the global flow results are presented. Particularly, the main focus of this contribution is on the behavior of the streamwise vortices. The vortex system is analyzed in detail and the effect on the high-lift behavior of the configuration is shown.

\subsection{Convergence Behavior}

For the simulations presented in this contribution, the major criterion to decide whether convergence is reached is the behavior of the aerodynamic coefficients. After a transient period for each angle of attack an oscillating behavior around a constant value with a limited amplitude is observed for both turbulence models. Nevertheless, the fluctuations are smaller for the Menter-SST simulations. When this behavior is observed, the normalized density residual is in a range between $10^{-4}$ and $10^{-3}$ and no further decrease is obtained. This indicates an unsteady flow resulting from the complex flow phenomena of this configuration, namely separated flow on the Fowler flap, wingtip vortex and vortices at the slat and clean nose edges. To investigate the effect of the unsteady flow 
phenomena on the solution, an unsteady simulation (URANS) with the JHh-v2 model has been performed at a $=6^{\circ}$. Based on the results of the steady simulation, the timespan of six convective time steps $\left(\mathrm{t}_{\mathrm{conv}}=\mathrm{c}_{\text {ref }} / \mathrm{V}_{\infty}\right)$ was simulated. The history of the aerodynamic coefficients of both types of simulations is shown in Figure 3.
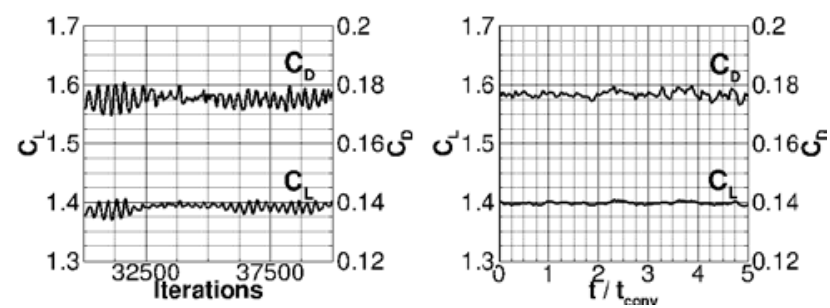

Fig. 3 History of aerodynamic coefficients of the JHh-v2 simulations at $\alpha=6^{\circ}$, left: steady simulation (last 10k iterations), right: unsteady simulation

It appears that the variation of the aerodynamic coefficients is lower for the unsteady simulation. Nevertheless, the level remains constant and is similar compared to the level predicted with steady simulations. It can be concluded that although the flow is unsteady, the results of the steady simulations are rather reliable in predicting the aerodynamic coefficients. The effect of steady and unsteady simulations on the prediction of the vortex system will be shown in section 3.3.

\subsection{Global Characteristics}

In this section, the aerodynamic performance and the global flow characteristics of the generic high-lift configuration are presented. Figure 4 shows the lift curve and the Lilienthal polar predicted with both turbulence models. It has to be mentioned that the presented values are averaged values of the last 5000 iterations of the steady simulations for each angle of attack.

The simulations with the Menter-SST turbulence model exhibit a linear behavior of the lift coefficient up to an angle of attack of $\alpha=12^{\circ}$. A further nonlinear increase is observed up to $\alpha=13.5^{\circ}$. At even larger angles of attack $\left(\alpha=14^{\circ}\right)$, no steady solution could be achieved, but the lift coefficient dropped down within the simulation. A transient solution illustrates large regions of separated flow downstream of the slat cut-off and on the inner part of the wing. Steady simulations with the $\mathrm{JHh}-\mathrm{v} 2$ turbulence model have also been performed up to $\alpha=13.5^{\circ}$. The lift coefficients are slightly larger compared to the MenterSST results up to $\alpha=10^{\circ}$. At higher incidences, the lift is lower. This behavior is explained by the pressure distributions. Figure 5 illustrates pressure distributions on the flap at $\alpha=10^{\circ}$ and $\alpha=12.5^{\circ}$.
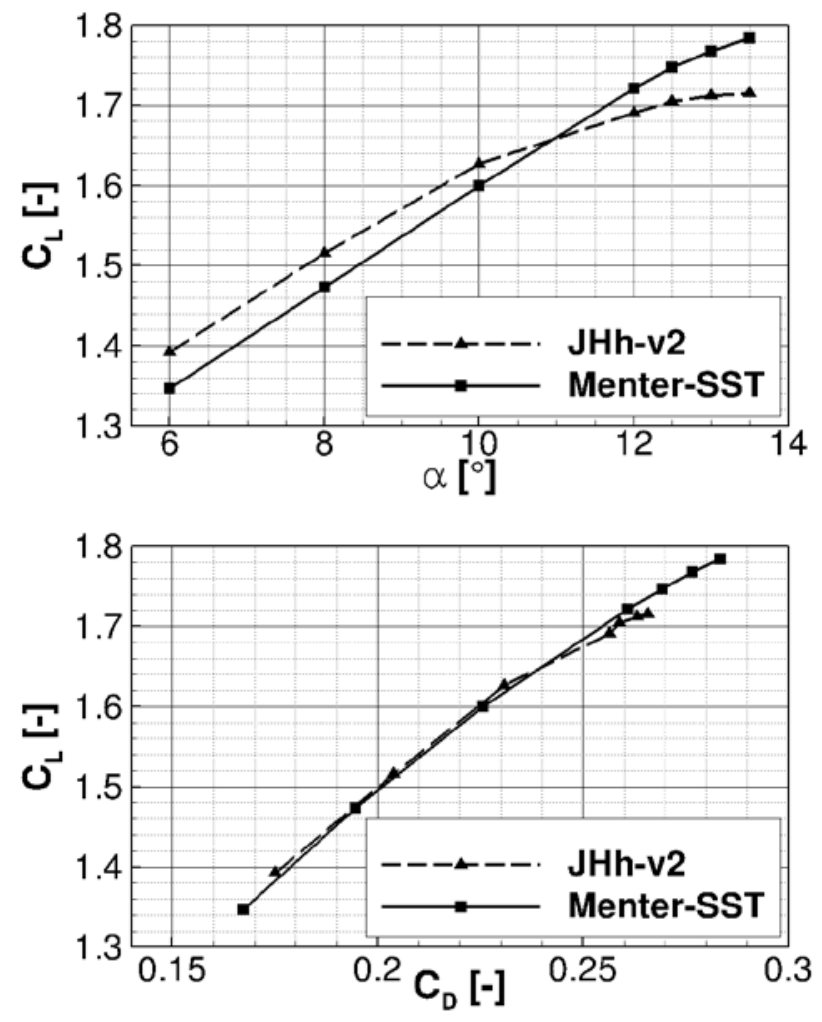

Fig. 4 Aerodynamic coefficients predicted with Menter-SST and JHh-v2 turbulence models, top: lift curve, bottom: Lilienthal polar
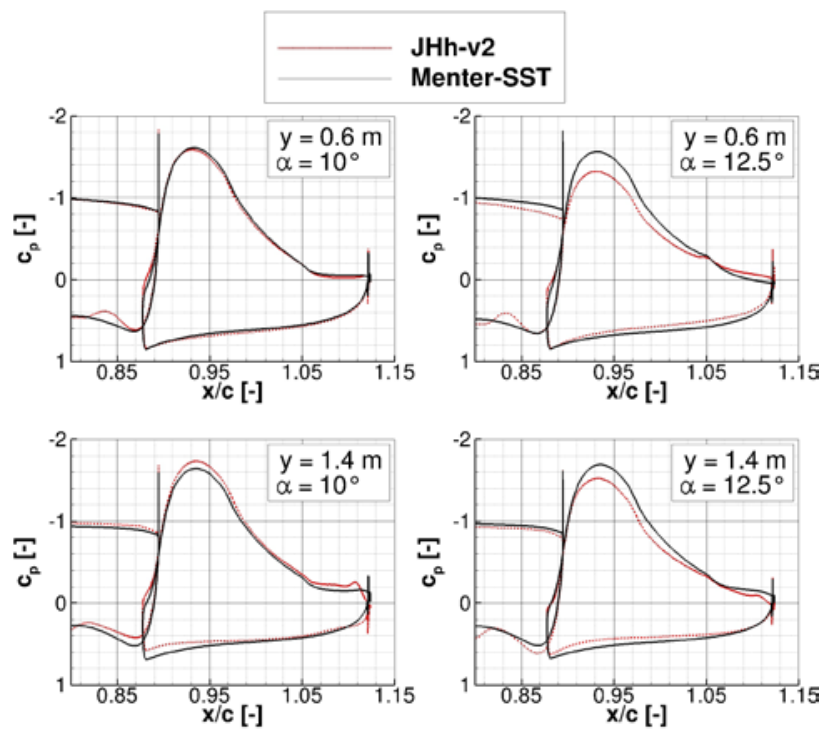

Fig. 5 Pressure distributions on the fowler flap at two spanwise positions, left: $\alpha=10^{\circ}$, right: $\alpha=12.5^{\circ}$

At $\alpha=10^{\circ}$ the pressure distributions of the Menter-SST and JHh-v2 models at an inboard position $(y=0.6 \mathrm{~m}$, two elements) are almost equal. At the outboard position $(y=1.4 m$, three elements) the suction peak is predicted slightly stronger with the JHh-v2 model. The same behavior is observed for the main element (not shown). Hence, a larger lift coefficient is predicted with the JHh-v2 model at $\alpha=10^{\circ}$. In contrast, the suction peaks over the whole span at $\alpha=12.5^{\circ}$ are weaker compared to the Menter-SST model, resulting in a lower lift coefficient. Another effect becomes apparent at the rear part of the 
flap. At $\alpha=10^{\circ}$ the pressure is almost constant at the downstream third of the flap, indicating a flow separation. At $\alpha=12.5^{\circ}$ the pressure further recovers towards the trailing edge. The behavior of the separation on the flap will be discussed later.

Although the lift curve differs between both turbulence models, the ratio of lift and drag is predicted similarly, resulting in a good agreement of the Lilienthal polars. Nevertheless, it has to be mentioned that the fluctuations of lift and drag are increased in the simulations at $\alpha=13^{\circ}$. $13.5^{\circ}$. To receive reliable results near maximum lift conditions, unsteady simulations at this angles of attack are in progress. Hence, the following evaluation of the global flow field and the behavior of the vortex system will focus on $\alpha=6^{\circ}$ (low, linear), $\alpha=10^{\circ}$ (medium, linear) and $\alpha=12.5^{\circ}$ (high, pre-stall).

To illustrate different flow effects that occur at the generic high-lift configuration, figure 6 shows wall streamlines and the skin friction coefficient in x-direction for the abovementioned angles of attack for both turbulence models.

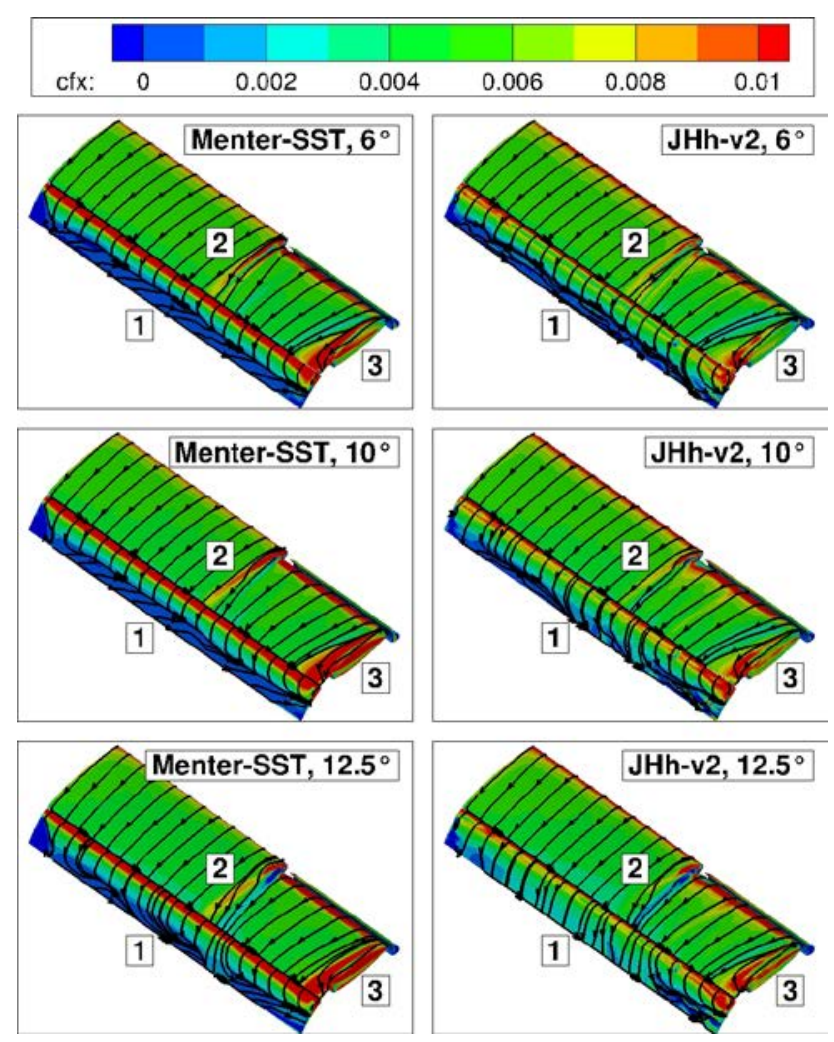

Fig. 6 Wall streamlines and skin friction distribution (x-direction) on the upper side of the generic high-lift configuration at different angles of attack

Figure 6 reveals different flow phenomena, which occur on the high-lift configuration. An important flow feature is the flow on the Fowler Flap. While the flow on the main wing and the slat is attached for the considered incidences, separated flow becomes visible on the rear part of the Fowler flap (1). The size and the location of the separated flow depend on the angle of attack and the turbulence model. At $\alpha=6^{\circ}$ both turbulence models show separated flow along the whole span. The Menter-SST turbulence model predicts a separation at about the last $40 \%$ of the flap, whereas the size is slightly smaller with the JHh-v2 model. Both turbulence models exhibit a decreasing size of the separation with increasing angle of attack. At $\alpha=12.5^{\circ}$, using the Menter-SST model, a small part of the flap downstream of the slat cut-off shows fully attached flow. At the rest of the flap only small regions show separated flow. With the JHh-v2 model, nearly the whole flow on the flap is attached.

Another remarkable flow effect occurs at the tip of the wing. At this location, the wall streamlines are curved inwards, representing the footprint of the wingtip vortex (3). At higher angles of attack, the values of the skin friction coefficient (x-direction) become larger in the influenced region due to a stronger tip vortex. For all incidences the wingtip vortex is a local phenomenon and does not considerably affect other parts of the configuration. Since this vortex is of no relevance for this contribution, no further evaluation will be performed. In contrast, the streamwise vortices arising at the edges of the slat and the clean nose are of great interest. These vortices likewise create distinct footprints downstream of the slat cut-off (2). The behavior of this vortex system varies depending on the turbulence model and the angle of attack. A detailed evaluation of the characteristics of the vortices is performed in the next part of this contribution.

\subsection{Characteristics of Streamwise Vortices}

At the generic high-lift configuration a system consisting of streamwise vortices develops and proceeds along the suction side. The development of these vortices is illustrated in figure 7 .

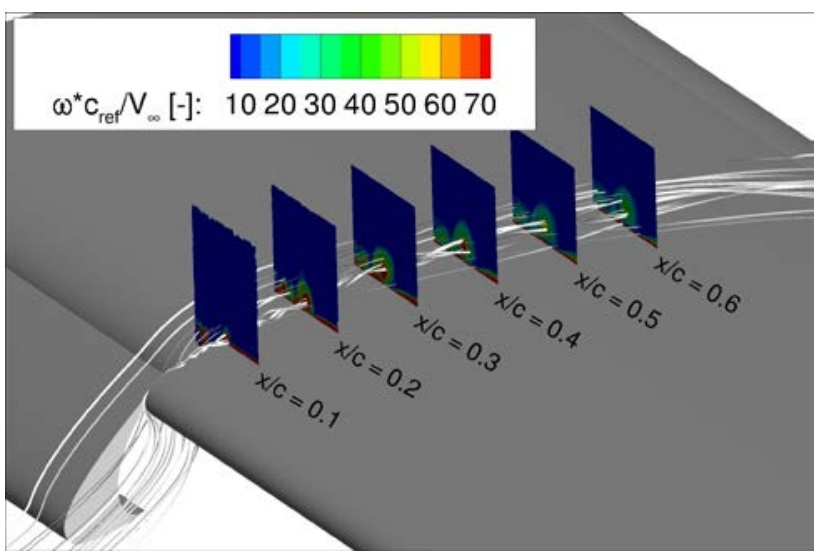

Fig. 7 Development of vortex system on the suction side of the high-lift wing. Contour plots of nondimensional vorticity at different positions on the wing simulated with Menter-SST model at $\alpha=6^{\circ}$.

It becomes apparent that two vortices arise. The first vortex develops at the edge of the spanwise cut off slat (slat vortex). At the edge of the clean nose, a compensating flow occurs due to high pressure differences between the suction and the pressure side. This flow generates another vortex, which proceeds on the suction side of the high-lift configuration (clean nose vortex). For further evaluation of these vortices slices at different chordwise positions will be evaluated. The slices with their corresponding position on the wing are also shown in figure 7 . 
In the first step, the influence of the computational grid on the prediction of the vortices is investigated. For the griddependence tests, three grids with a different grid resolution within the vortex region are used (details in 2.3.). Figure 8 shows results of the simulations with the Menter-SST model on the different grids at $\alpha=10^{\circ}$.

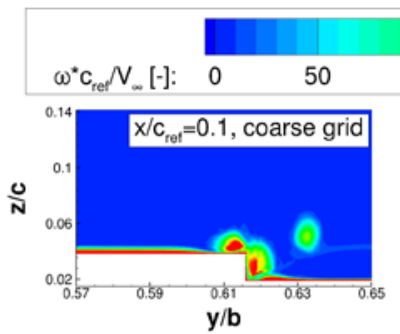

$100 \quad 150 \quad 200 \quad 250$
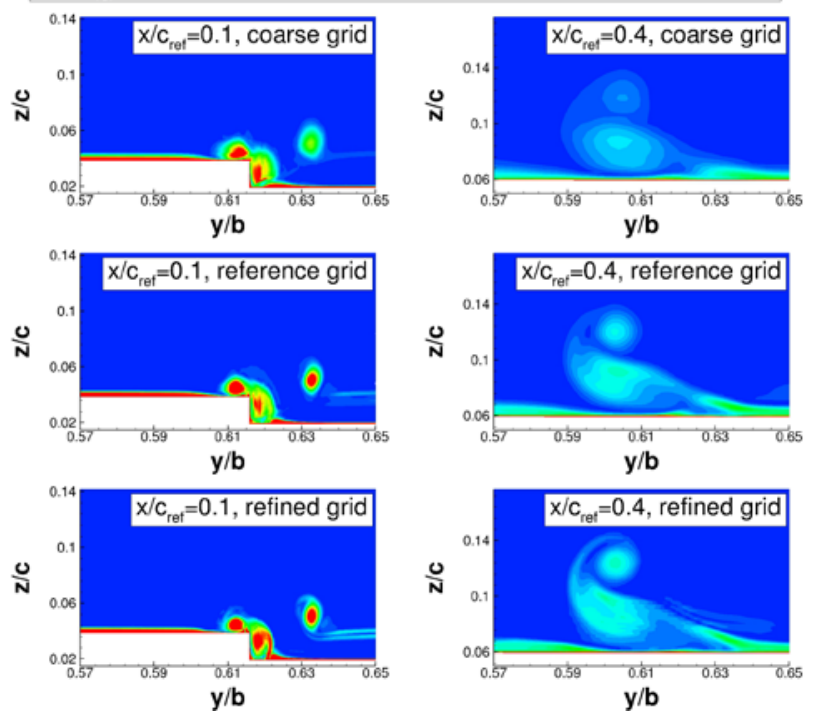

Fig. 8 Nondimensional vorticity at different chordwise positions simulated with the Menter-SST model at $\alpha=10^{\circ}$ with different grids

Significant differences in the prediction of the vortices occur between the coarse and the reference grid. At a chordwise position of $x / c_{\text {ref }}=0.1$ the slat vortex is predicted clearly stronger on the reference grid. However, the clean nose vortex is predicted in a similar manner on both grids at this position. Further downstream $\left(x / c_{\text {ref }}=0.4\right)$, both vortices dissipate rapidly on the coarse grid. The structure and the strength differ from the vortices predicted on the reference grid. Obviously, the coarse grid is not able to predict the vortices accurately. In contrast, the differences between the reference grid and the locally refined grid are much smaller. The vortices predicted on both grids show the same structure and the same strength at both chordwise positions. Although the resolution has been quadrupled for the locally refined grid, almost no effect on the prediction of the vortices is visible. Hence it can be concluded that the spatial resolution of the vortices is sufficient on the reference grid. For this reason, the following results arise from simulations on the reference grid.

In section 3.1 it has been shown that fluctuations of the aerodynamic coefficients appear within the steady simulations. Hence, the influence of the solver type (steady respectively unsteady simulation) on the prediction of the vortices is analyzed. Therefore, the steady solution and a time-averaged solution with the unsteady solver at two different chordwise positions simulated with the JHh-v2 model are shown in figure 9 for an angle of attack of $\alpha=6^{\circ}$. The averaging has been performed for one convective time step between $\mathrm{t} / \mathrm{t}_{\mathrm{conv}}=$ 5 and $\mathrm{t} / \mathrm{t}_{\mathrm{conv}}=6$.
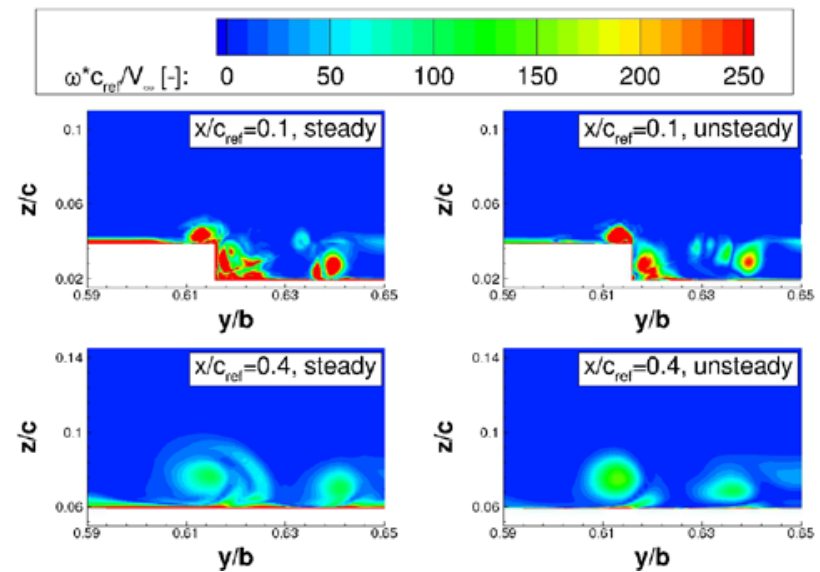

Fig. 9 Contour plots of nondimensional vorticity at two chordwise positions (JHh-v2 turbulence model, $\left.\alpha=6^{\circ}\right)$, left: steady simulation, right: unsteady simulation (time-integrated between $t / t_{c o n v}=5-6$ )

It arises that the vortices predicted with the steady and the unsteady solver are similar. Both types of simulation show vortices of about the same size at the both slices. The most noticeable difference between the solutions occurs at a chordwise position of $x / c_{\text {ref }}=0.1$. At this point, the slat vortex predicted with the unsteady solver is slightly weaker compared to the steady solver. Nevertheless, the size, the position and the appearance of the slat vortex is predicted similarly. In addition, the steady and unsteady solvers show secondary vortices in the region of the slat vortex. The appearance and strength of these vortices slightly differs between both solvers. At the slice located downstream $\left(x / c_{\text {ref }}=0.4\right)$, the differences between both solutions are smaller. The steady and unsteady solver predict vortices of almost the same size, strength and position. Although slight differences occur at both chordwise positions, it can be concluded that the steady solver captures the important effects of the vortex system, namely the strength, position and the size. For this reason, the results of the steady simulations with the Menter-SST and JHh-v2 turbulence models are used to analyze the influence of turbulence model and the incidence on the behavior of the vortex system. Nevertheless, it has to be noted that these results come from unconverged solutions, which still show oscillations in the aerodynamic coefficients (q.v. 3.1). Figure 10 shows the development of the vortex system at $\alpha=6^{\circ}$ for both turbulence models. 

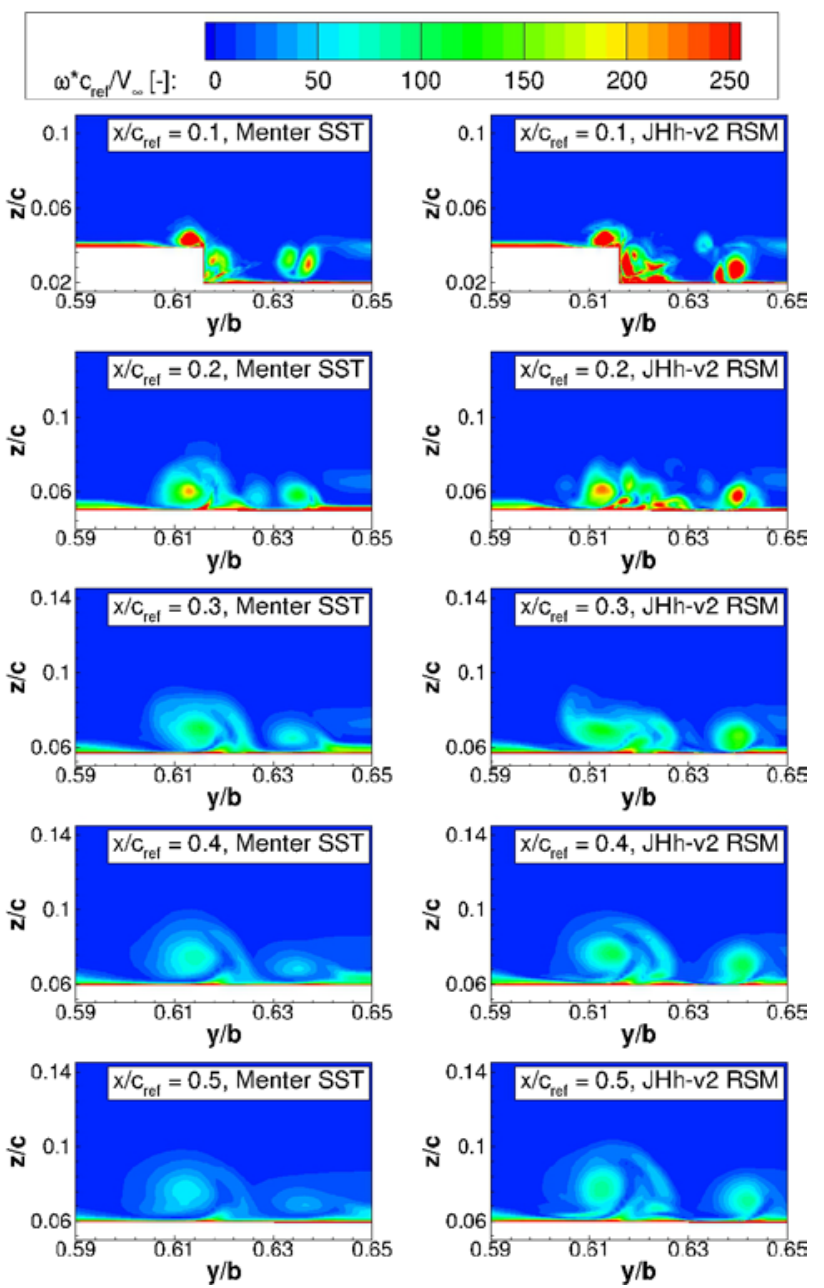

Fig. 10 Nondimensional vorticity at different chordwise positions at $\alpha=6^{\circ}$, left: Menter-SST turbulence model, right: JHh-v2 RSM

At the first presented position at $x / c_{\text {ref }}=0.1$, different regions of increased nondimensional vorticity become apparent. Both turbulence models show distinct regions of increased vorticity between $y / b=0.63-0.64$. At this angle of attack, the aerodynamic loads of the slat are very low and different vortical structures arise at the edges of the slat. Furthermore, the formation of vortical structures through the flow around the clean nose is observed $(\mathrm{y} / \mathrm{b}=$ 0.61 - 0.62). While proceeding downstream, the vortical structures behind the slat merge. The same holds for the structures behind the clean nose. Finally, at a chordwise position of $x / c_{\text {ref }}=0.3$, two distinct vortices have formed. The positions $\mathrm{x} / \mathrm{c}_{\text {ref }}=0.4$ and $\mathrm{x} / \mathrm{c}_{\text {ref }}=0.5$ reveal a further decrease of the maximum nondimensional vorticity with a simultaneous widening of both vortices. The spanwise position $(y / b)$ and the position above the suction side of the wing remain nearly constant. This behavior and the appearance of the vortex system is predicted by both turbulence models. Nevertheless, the results differ between the Menter-SST and the JHh-v2 turbulence model regarding the strength of the vortices. The JHh-v2 model predicts significant stronger vortices already closely behind the origin. Furthermore, the $\mathrm{JHh}-\mathrm{v} 2$ model is able to conserve the vortices for a longer distance. This behavior is also illustrated in figure 11 , which visualizes the vortices using iso-surfaces of the non-dimensional vorticity.
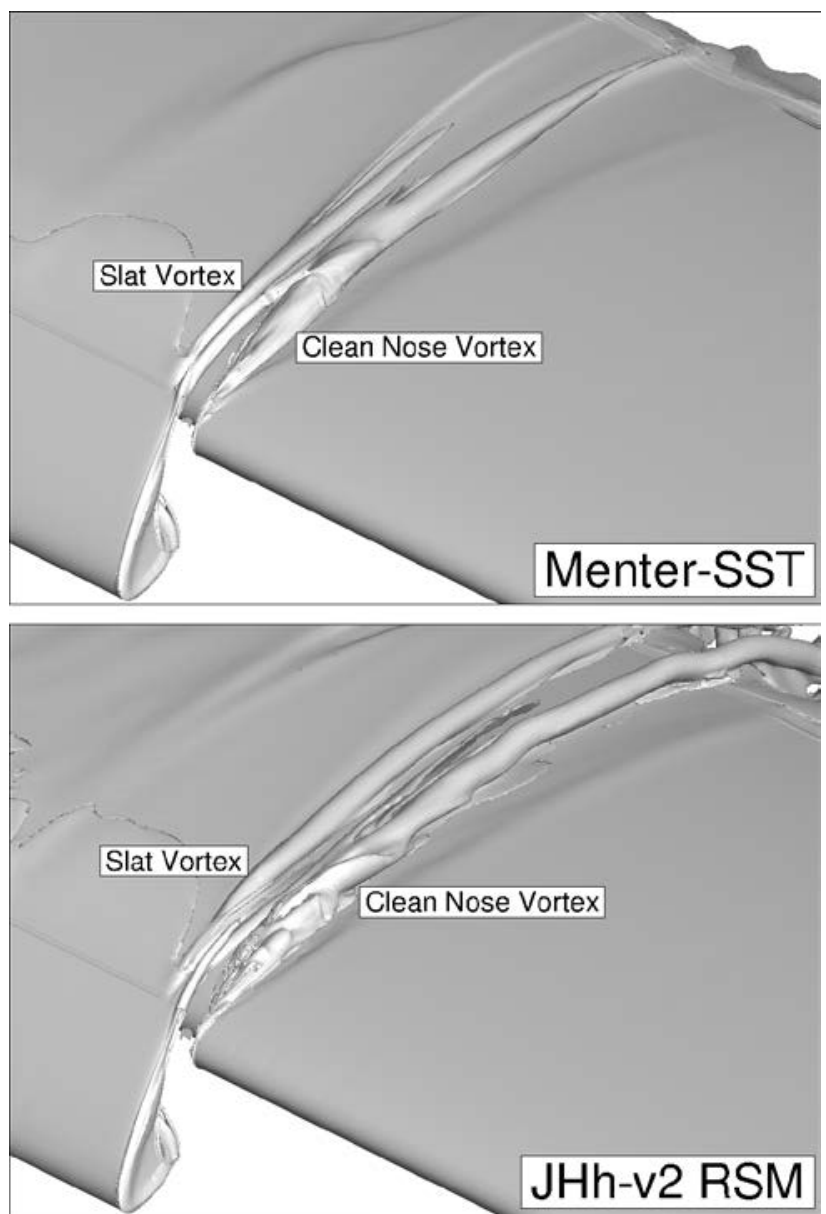

Fig. 11 Iso-Surfaces of Nondimensional Vorticity $\omega^{*} \mathrm{c}_{\mathrm{ref}} / \mathrm{V}_{\infty}=35$ at $\alpha=6^{\circ}$, top: Menter-SST, bottom: JHh-v2

Figure 11 clearly illustrates the formation of the vortices at the edges and the development along the suction side of the wing. This visualization reveals the conservation of the vortices with the JHh-v2 model, since the iso-surfaces proceed along the whole suction side. This result is in good agreement with the results of Craft [13] and Cécora [14], who also observed the effects of stronger vortices and a longer conservation with Reynolds-Stress-Models compared to eddy viscosity models.

At an angle of attack of $\alpha=6^{\circ}$, no interaction between the slat vortex and the clean nose vortex is observed. A different situation appears at $\alpha=10^{\circ}$, which is illustrated in figure 12 . 

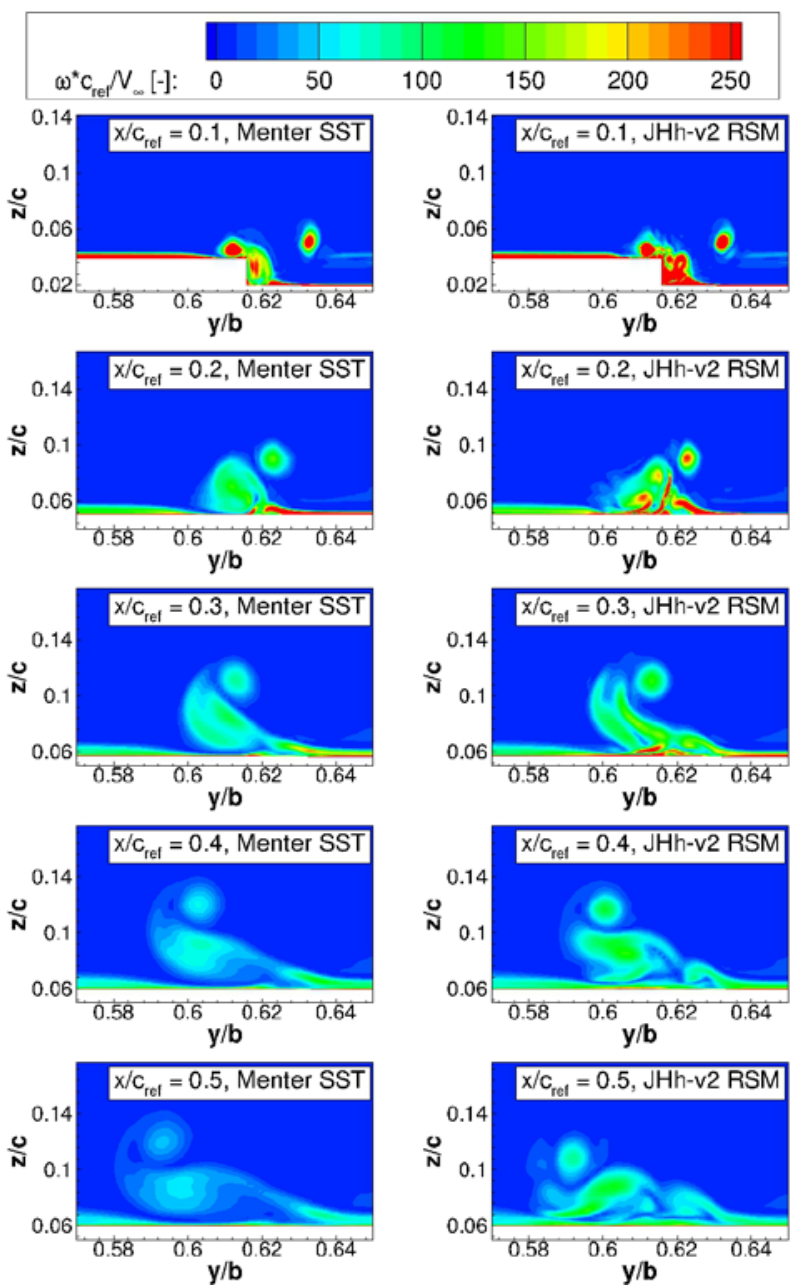

Fig. 12 Nondimensional chordwise positions at $\alpha=10^{\circ}$, left: Menter-SST turbulence model, right: JHh-v2 RSM

It becomes apparent that the mechanism of the formation of the clean nose vortex is similar. However, the appearance of the slat vortex differs. A distinct circular region of increased vorticity already appears at a chordwise position of $\mathrm{x} / \mathrm{c}_{\mathrm{ref}}=0.1$ due to $\mathrm{a}$ higher aerodynamic load of the slat at this incidence. This vortex is slightly away from the surface. While proceeding downstream, this vortex is drifting inwards without changing the appearance. At $x / c_{\text {ref }}=0.3$ the slat vortex interacts with the clean nose vortex. The clean nose vortex is displaced by the slat vortex and a change of the appearance of the clean nose vortex is observed. Further downstream, this behavior continues. Although the general behavior is captured by both turbulence models, larger differences between the Menter-SST and the JHhv2 turbulence model emerge. The results of the MenterSST turbulence model show quite weak vortices at a position of $\mathrm{x} / \mathrm{c}_{\mathrm{ref}}=0.3$ due to the rapid decay of the strength. Hence, the interaction between the slat and the clean nose vortex is smaller compared to the JHh-v2 model, which can be observed most clearly at a position $\mathrm{x} / \mathrm{c}_{\text {ref }}=0.5$. At this position, the vortices predicted with the Menter-SST model have already lost most of the strength and the slat vortex is nearly above the remaining part of the clean nose vortex. Compared to the Menter-SST model the $\mathrm{JHh}-\mathrm{v} 2$ model predicts stronger vortices at this position. In addition, the position of the vortices relative to each other changed. The slat vortex is at an inboard position, while the remaining vortical structures of the clean nose vortex are on the outer side. This behavior is again illustrated in figure 13.
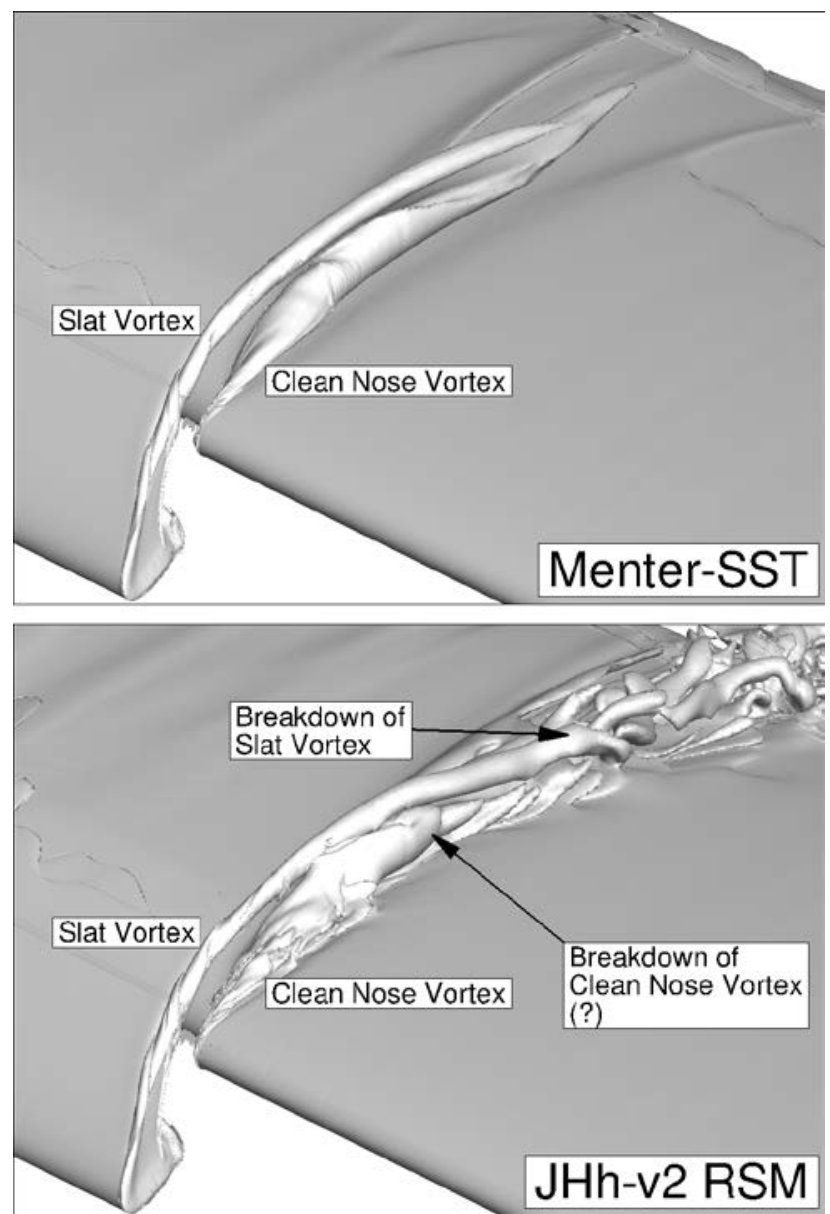

Fig. 13 Iso-Surfaces of Nondimensional Vorticity $\omega^{*} c_{\text {ref }} / V_{\infty}=35$ at $\alpha=10^{\circ}$, top: Menter-SST, bottom: JHh-v2

The iso-surfaces of nondimensional vorticity demonstrate the differences between the prediction of the vortex system of the Menter-SST and the JHh-v2 turbulence model. The weak interaction predicted with the MenterSST model at $x / c_{\text {ref }}=0.3$ becomes visible. Although the clean nose vortex is displaced by the slat vortex, the weak vortex withstands the displacement. In contrast, the results of the $\mathrm{JHh}-\mathrm{v} 2$ model expose a different behavior of the interaction. When both vortices interact, the clean nose vortex changes its appearance. Due to the displacement of the slat vortex, the clean nose vortex spreads out in the spanwise direction and decomposes. This decomposition takes place in a region of an adverse pressure gradient on the suction side of the high-lift configuration, which favors a breakdown of the vortex. It is not clearly determined if the adverse pressure gradient or the influence of the slat vortex is primarily responsible for the decomposition of the clean nose vortex. For this reason, a vortex breakdown in the classical sense is questionable and therefore marked with a (?) in figure 13. Another effect is observed for the slat vortex. The appearance of this vortex is constant up to a chordwise position of $x / c_{\text {ref }}=0.5$. Nevertheless, figure 13 shows a changing behavior downstream. Still, at this position an adverse pressure gradient is present. The slat vortex breaks down in a spiral movement. At positions further downstream, decomposed structures of increased vorticity 
are present.

Compared to $\alpha=10^{\circ}$, the behavior of the vortex system at $\alpha=12.5^{\circ}$ exhibits strong similarities. The contour plots of nondimensional vorticity are presented in figure 14 .
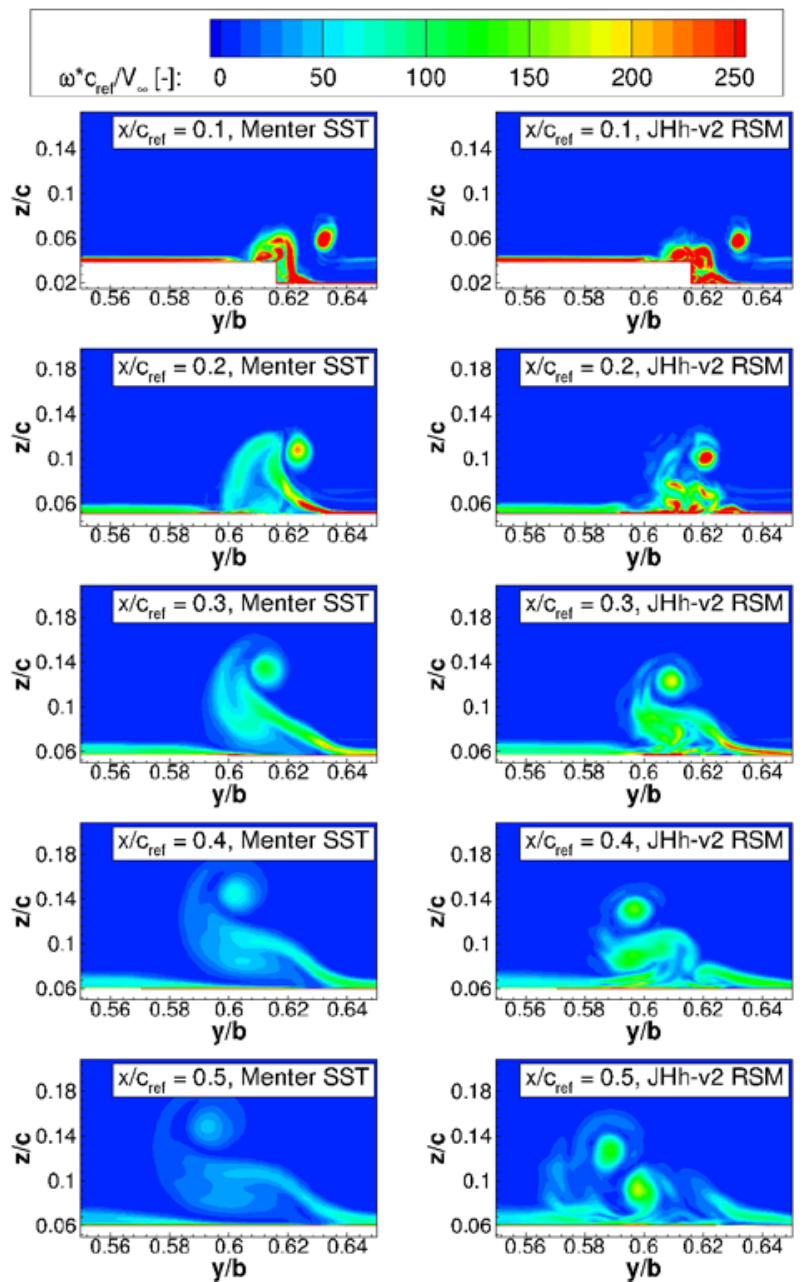

Fig. 14 Nondimensional vorticity at different chordwise positions at $\alpha=12.5^{\circ}$, left: Menter-SST turbulence model, right: JHh-v2 RSM

It appears that the mechanism of the formation of the slat and the clean nose vortex at $\alpha=12.5^{\circ}$ is similar compared to $\alpha=10^{\circ}$. Nevertheless, the strength of both vortices at $\mathrm{x} / \mathrm{c}_{\mathrm{ref}}=0.1$ is higher due to higher aerodynamic loads at this angle of attack. Furthermore, the interaction between both vortices occurs earlier. At $x / c_{\text {ref }}=0.2$, the Menter-SST model shows a widened clean nose vortex, which is already influenced by the slat vortex. The JHh-v2 model likewise reveals an interaction of the vortices, but in contrast to the Menter-SST model, the clean nose vortex already decomposes. Spots of increased vorticity are visible at this position. Further downstream the interaction predicted with the Menter-SST model is similar to $\alpha=10^{\circ}$. The interaction between the vortices is dominated by a displacement of the clean nose vortex. A decomposition or breakdown is not observed. In contrast, the interaction predicted with the JHh-v2 model is of a fundamentally different character. Downstream of the observed decomposition of the clean nose vortex, a reformation of this vortex occurs. Between $x / c_{\text {ref }}=0.4-0.5$, a nearly circular region has formed again. Compared to upstream positions, the maximum nondimensional vorticity is increased again. To illustrate this effect, iso-surfaces of nondimensional vorticity are shown in figure 15.
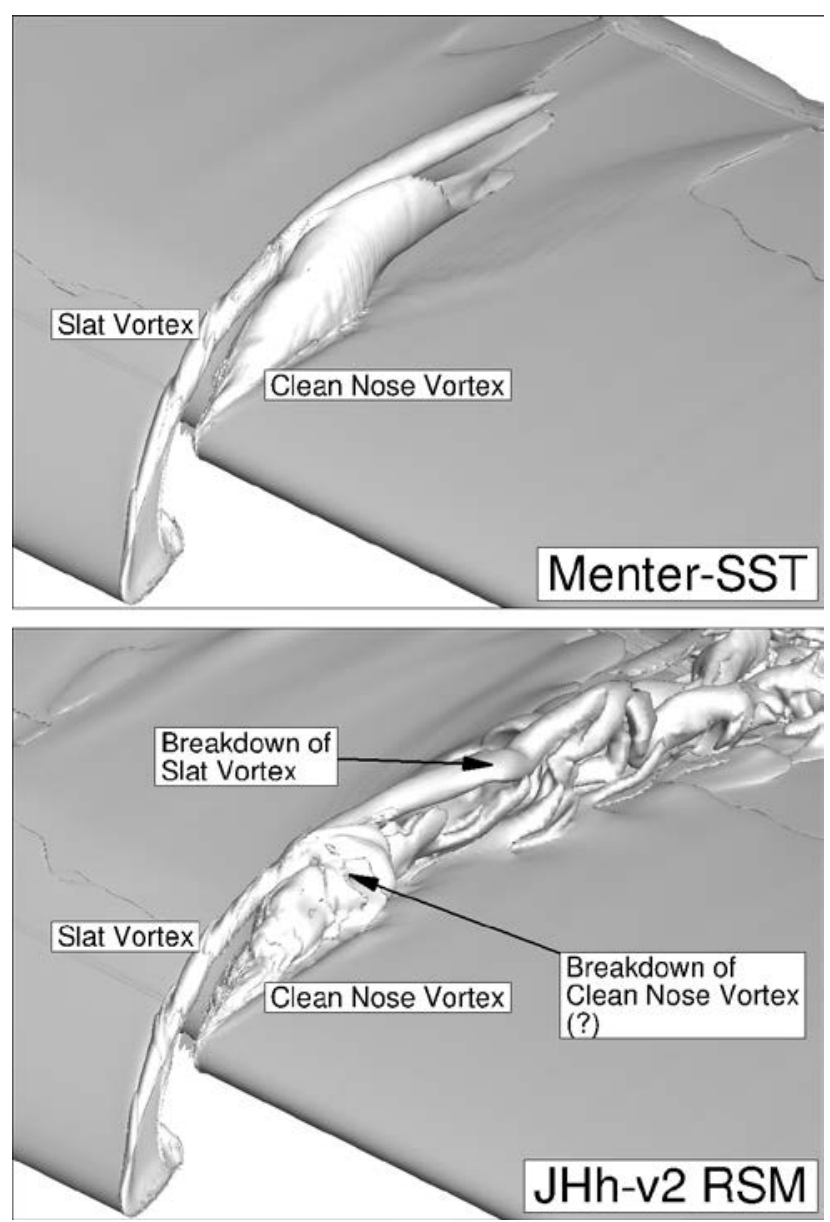

Fig. 15 Iso-Surfaces of Nondimensional Vorticity $\omega^{*} c_{\text {ref }} / V_{\infty}=35$ at $\alpha=12.5^{\circ}$, top: Menter-SST, bottom: JHh-v2

For both turbulence models a widened clean nose vortex is visible. Again, the visualization of the vortex system predicted with the Menter-SST model shows a displacement of the clean nose vortex without decomposition or breakdown. The results of the JHh-v2 model show the displacement of the clean nose nose vortex at an earlier position compared to $\alpha=10^{\circ}$. The reason for this is the strong interaction with the slat vortex, which occurs at an upstream location. Furthermore, the adverse pressure gradient is stronger at higher incidences. This effect is also responsible for the breakdown of the slat vortex, which again is observed at an upstream position compared to $\alpha=10^{\circ}$. The visualization of the vortices also demonstrates that distinct vortical structures reformate after the clean nose vortex decomposes.

At $\alpha=12.5^{\circ}$, regions of a negative skin friction coefficient occur close behind the edge of the clean nose (compare figure 4). Here the upwards directed velocity components of the outboard side of the clean nose vortex destabilize the boundary layer. Local limited regions of separated flow occur. This effect is stronger for the Menter-SST model, where the clean nose vortex is only displaced and not decomposed. Further downstream, a stronger effect is observed for the JHh-v2 model. Here, the clean nose vortex reformates and influences the boundary layer. The 
Menter-SST model is not able to preserve the strength of the vortex and thus, the effect on the boundary layer is lower. Nevertheless, the influenced region behind the cutoff is small for both turbulence models and for that reason the overall aerodynamic behavior of the high-lift configuration at this angle of attack is not influenced significantly.

\section{CONCLUSION}

Results of flow simulations of streamwise vortices on a generic three-dimensional high-lift configuration at different angles of attack have been presented. The simulations were performed with the DLR TAU-Code as flow solver applying the Menter-SST eddy viscosity and the JHh-v2 RSM turbulence model. Two vortices develop at the edges of the slat and the clean nose, proceeding downstream on the suction side of the wing.

Steady simulations expose slight fluctuations of the aerodynamic coefficients. However, a comparison with an unsteady simulation shows that the results produced with a steady solver are similar concerning the level of the aerodynamic coefficients and the behavior of the vortices. Nevertheless, at incidences close to stall it becomes necessary to perform unsteady simulations

The results at all considered angles of attack reveal a different behavior of the vortices for both turbulence models. In general, the vortices predicted with the JHh-v2 model are stronger compared to the Menter-SST model. In addition, a strong decay of the vortices is observed for the Menter-SST turbulence model. The JHh-v2 RSM model preserves the vortices better while proceeding downstream. This observation corresponds to results from the literature [13][14]. Furthermore, differences between the Menter-SST and the JHh-V2 model occur concerning the interaction of the vortices. At $\alpha=6^{\circ}$, no interaction is observed for both turbulence models. In contrast, at $\alpha=10^{\circ}$ and $\alpha=12.5^{\circ}$ the slat vortex drifts inwards and displaces the clean nose vortex. For this angles of attack, the results of the JHh-v2 model exhibit a decomposition of the clean nose vortex when the interaction takes place. This decomposition is not observed with the Menter-SST model, where the clean nose vortex is simply deformed. Furthermore, at downstream positions, the JHh-v2 model predicts a breakdown of the slat vortex. Again, this effect is not captured by the Menter-SST model.

The vortex behavior influences the boundary layer on the suction side of the wing. The wall streamlines are curved by the vortices close to the surface. Particularly, at high angles of attack differences between the Menter-SST and the JHh-v2 model become visible. The reason for this is the behavior of the interacting vortices. Here, the different structures of the predicted interaction are represented at the skin friction distribution. To identify the effect of the vortex interaction and breakdown on the stall behavior of the high-lift configuration, unsteady simulations at the corresponding angles of attack have to be performed, which will be part of future research within this project. Further plans of future work include an Improved Delayed Detached Eddy Simulation (IDDES). This simulation wil show whether a RANS simulation can capture the important flow mechanisms or whether a scale-resolving simulation is required to capture the complex flow features. In addition, a wind-tunnel experiment with the presented configuration is planned. These experiments will deliver a data base for a comparison and allow a quantitative evaluation of the simulations presented in this contribution.

\section{ACKNOWLEDGMENTS}

The authors wish to acknowledge the German Federal Ministry for Economic Affairs and Energy (BMWi) for funding this research activity (grant number: 20A1301B). The views and conclusions contained herein are those of the authors and should not be interpreted as necessarily representing the official policies or endorsements. Furthermore, the authors would like to thank the NorthGerman Supercomputing Alliance (HLRN) for providing computational resources within the project nii00091.

\section{Gefördert durch:}

\section{Bundesministerium für Wirtschaft und Energie}

\section{aufgrund eines Beschlusses des Deutschen Bundestages}

\section{REFERENCES}

[1] Rudolph, P. K. C., High-Lift Systems on Commercial Subsonic Airliners, NASA CR 4746, 1996.

[2] Smith, A. M. O., High Lift Aerodynamics, Journal of Aircraft 12, No. 6:501-530, 1975.

[3] Haines, A. B., Young, A. D., Scale Effects on Aircraft and Weapon Aerodynamic, AGARDograph 323, pp. $27-65,1994$

[4] Eliasson, P., Catalano, P, Le Pape, M.-C., Ortmann, J., Pelizzari, E. and Ponsin, J., Improved CFD Predictions for High Lift Flows in the European Project EUROLIFT II, AIAA 2007-4303, Miami, 2007.

[5] Frhr. v. Geyr, H., Schade, N., v. d. Burg, J. W. Eliasson, P. and Esquieu, S., CFD Prediction of Maximum Lift Effects on Realistic High-LiftCommercial-Aircraft-Configurations within the European project EUROLIFT II, AIAA 2007-4299, Miami, 2007.

[6] Rudnik, R. and Frhr. v. Geyr, H., The European High Lift Project EUROLIFT II - Objectives, Approach, and Structure, AIAA 2007-4296, Miami, 2007.

[7] Rudnik, R., Stall Behavior of the EUROLIFT High Lift Configurations, AIAA 2008-836, Reno, 2008.

[8] Wild, J., Brezillon, J., Amoignon, O., Quest, J., Moens, F. and Quagliarella, D., Advanced High-Lift Design by Numerical Methods and Wind Tunnel Verification within the European Project EUROLIFT II, AIAA 2007-4300, Miami, 2007.

[9] Rudnik, R., Reckzeh, D. and Quest, J., HINVA - High lift INflight Validation - Project Overview and Status, AIAA 2012-0106, Nashville, 2012. 
[10] Bier, N., Rohlmann, D. and Rudnik, R., Numerical Maximum Lift Prediction of a Realistic Commercial Aircraft in Landing Configuration, AIAA 2012-0279, Nashville, 2012.

[11] Sclafani, A. J., Slotnick, J. P., Vassberg, J. C., Pulliam, T. H. and Lee, H. C., OVERFLOW Analysis of the NASA Trap Wing Model from the First High Lift Prediction Workshop, AIAA 2011-866, Orlando, 2011.

[12] Crippa, S., Melber-Wilkending, S. and Rudnik, R., DLR Contribution to the First High Lift Prediction Workshop, AIAA 2011-938, Orlando, 2011.

[13] Craft, T. J., Gerasimov, A. V., Launder, B. E. and Robinson, C. M. E., A computational study of the near-field generation and decay of wingtip vortices, International Journal of Heat and Fluid Flow 27, 684695, 2006.

[14] Cécora, R.-D., Radespiel, R., Eisfeld, B. and Probst, A., Differential Reynolds-Stress Modeling for Aeronautics, Journal of Aircraft, Vol. 53, No. 3, pp. 739-755, 2015.

[15] Landa, T., Wild, J. and Radespiel, R., Simulation of Longitudinal Vortices on a High-Lift Wing, Radespiel, R. et al (Eds): Advances in Simulation of Wing and Nacelle Stall, Notes on Numerical Fluid Mechanics and Multidisciplinary Design, Vol. 131, 351-366, Springer, ISBN 978-3-319-21126-8, 2015.

[16] Schwamborn, D., Gerhold, T. and Heinrich, R., The DLR TAU-Code: Recent Applications in Research and Industry, ECCOMAS CFD 2006, Egmond aan Zee, 2006.

[17] Schwamborn, D., Gardner, A. D., von Geyr, H., Krumbein, A., Lüdeke, H. and Stürmer, A., Development of the DLR TAU-Code for Aerospace Applications, ICASAT 2008, Bangalore, 2008.

[18] Menter, F. R., Zonal Two Equation k- $\omega$ Turbulence Models for Aerodynamic Flows, AIAA 93-2906, Orlando, 1993.

[19] Jakirlic, S. and Hanjalic, K., A new approach to modelling near-wall turbulence energy and stress dissipation, Journal of Fluid Mechanics, 459:139-166, 2002.

[20] Probst, A. and Radespiel, R., Implementation and Extension of a Near-Wall Reynolds-Stress Model for Application to Aerodynamic Flows on Unstructured Meshes, AIAA 2008-770, Reno, 2008.

[21] Reuß, S., Probst, A. and Knopp, T., Numerical investigation of the DLR F15 two-element airfoil using a Reynolds stress model, Third Symposium "Simulation of Wing and Nacelle Stall", Braunschweig, 2012.

[22] Wild, J., Mach and Reynolds Number Dependencies of the Stall Behavior of High-Lift Wing-Sections, Journal of Aircraft, Vol. 50, No. 4, pp. 1202-1216, 2013.

[23] Hahn, D., Scholz, P. and Radespiel, R., Experimental evaluation of the stall characteristics of a two-element high-lift airfoil, Second Symposium "Simulation of Wing and Nacelle Stall", Braunschweig, 2010.

[24] Hühne, C.-P., Mahmood, S., Scholz, P. and Radespiel, R., Active flow control at a swept wing: A comparison of numerical and experimental results, AIAA 2013-2798, San Diego, 2013.

[25] Büscher, A. and Radespiel, R., A method for the aerodynamic analysis and design of nonplanar lifting configurations at transonic speeds, Jahrbuch DGLR, Bd. 1:603-612, 2003.

[26] Probst, A., Radespiel, R. and Rist, U., LinearStability-Based Transition Modeling for Aerodynamic
Flow Simulations with a Near-Wall Reynolds-Stress Model. AIAA Journal 50(2), pp. 416-428, 2012.

[27] Cécora, R.-D., Reynolds-Spannungsmodell der Turbulenz für Anwendungen der Flugzeugaerodynamik, NFL Forschungsbericht 201527, Braunschweig, 2015. 